\title{
Polarization Pendellösung and the Generation of Circularly Polarized X Rays with a Quarter-Wave Plate
}

\section{Citation}

Golovchenko, J. A., B. M. Kincaid, R. A. Levesque, A. E. Meixner, and D. R. Kaplan. 1986.

Polarization Pendellösung and the Generation of Circularly Polarized X Rays with a QuarterWave Plate. Physical Review Letters 57, no. 2: 202-206. doi:10.1103/physrevlett.57.202.

\section{Published Version}

doi:10.1103/PhysRevLett.57.202

\section{Permanent link}

http://nrs.harvard.edu/urn-3:HUL.InstRepos:29407040

\section{Terms of Use}

This article was downloaded from Harvard University's DASH repository, and is made available under the terms and conditions applicable to Other Posted Material, as set forth at http:// nrs.harvard.edu/urn-3:HUL.InstRepos:dash.current.terms-of-use\#LAA

\section{Share Your Story}

The Harvard community has made this article openly available.

Please share how this access benefits you. Submit a story.

\section{Accessibility}




\title{
Polarization Pendellösung and the Generation of Circularly Polarized X Rays with a Quarter-Wave Plate
}

\author{
J. A. Golovchenko, B. M. Kincaid, R. A. Levesque, ${ }^{(a)}$ A. E. Meixner, ${ }^{(b)}$ and D. R. Kaplan \\ AT\&T Bell Laboratories, Murray Hill, New Jersey 07974 \\ (Received 9 April 1986)
}

\begin{abstract}
A continuously tunable $\mathrm{x}$-ray compensator that works in conjunction with synchrotron-radiation sources has been developed. The phenomena of polarization Pendellösung has been observed during its normal operation and the generation of circularly polarized $\mathrm{x}$ rays is verified through an observation of the magnetic Compton effect.
\end{abstract}

PACS numbers: $42.10 . \mathrm{Nh}$

Several recent investigations have dealt with the generation of polarized $\mathrm{x}$ rays by use of dynamical $\mathrm{x}$ ray diffraction techniques. ${ }^{1-4}$ Besides the inherently interesting $\mathrm{x}$-ray-crystal interactions demonstrated in these studies the prospect of extending the classical long-wavelength manifestations of crystal optics, (i.e., optical birefringence, rotary power, etc.) into the hard- $x$-ray region presents several new challenges and opportunities for experimental and theoretical physicists. An application of particular current interest lies in the ability to obtain spin-dependent momentum profiles of magnetic materials by examination of the Compton scattering of circularly polarized $x$ rays. ${ }^{5-7}$ Until now the only source of spin-polarized photons for such experiments has been radioactive decay and a new strong source, e.g., synchrotron radiation, would have a significant influence on such studies. However, present synchrotron-radiation sources provide mainly linearly polarized light. The possibility of providing circular polarization through the use of either helical wigglers $^{8}$ or tandem crossed wigglers ${ }^{9}$ is currently under discussion for the next generation of storage rings. External optics which would serve the same purpose without perturbing electron orbits or the expense of large magnetic structures would clearly be desirable.

We describe here a recent series of experiments using a synchrotron-radiation source and $\mathrm{x}$-ray compensator plate designed to isolate polarization Pendellösung in the plate and generate circularly polarized $\mathrm{x}$ rays at arbitrary energies. The Pendellösung or oscillation effect we observe is somewhat different in several respects from the one commonly known in diffraction physics ${ }^{10}$ and it is probably best introduced here simply by a description of the experimental setup for observing it.

Figure 1 is a schematic illustration of the experiment. $X$ rays from the Cornell high-energy synchrotron source operating at $5.2 \mathrm{GeV}$ and $12 \mathrm{~mA}$ in the colliding-beam mode were collected by an asymmetrically cut silicon crystal oriented to diffract from the (220) planes in the Bragg geometry. A few square millimeters of the white beam were intercepted. The asymmetry was such as to increase the angular accep- tance of the incident beam and reduce the angular divergence of the Bragg-diffracted emergent beam. Completely glacing incidence was obtained at $\sim 18$ $\mathrm{keV}$, which determined the highest energy of our experiments. Renninger has described the collimating properties of such an element. ${ }^{11}$ The collimated monochromatic beam thus obtained is then directed onto another silicon crystal also oriented to diffract from the (220) planes but now in the transmission Laue geometry, i.e., the diffracted beam propagates into the crystal. This crystal serves as the $\mathrm{x}$ ray com-

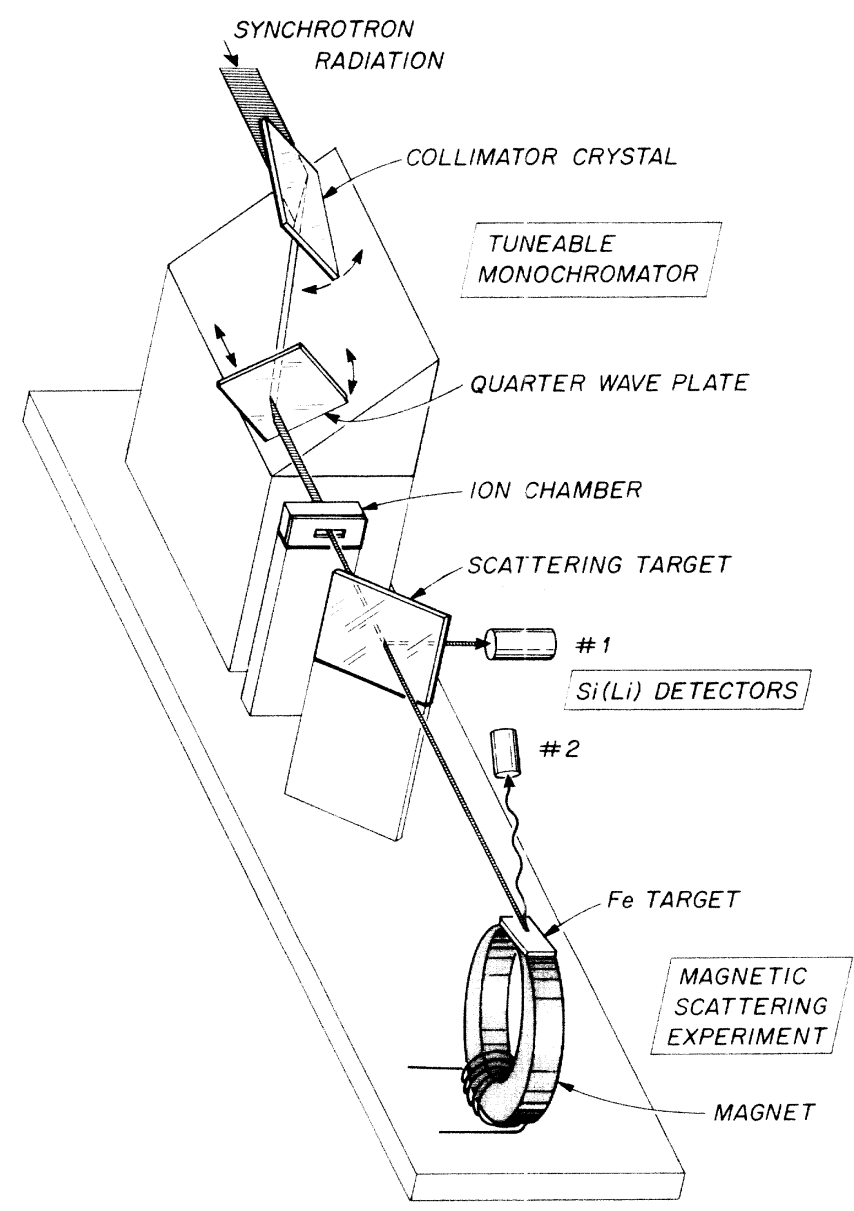

FIG. 1. Schematic layout of experiment. 
pensator and quarter-wave plate. The (220) planes are normal to the $\mathrm{x}$-ray entry surface and the thickness of the plate was chosen to be $1.39 \mathrm{~mm}$. The two crystals were mounted in a piezoelectric, feedback-stabilized, mechanical-linkage system ${ }^{12}$ capable of keeping the two sets of crystal planes parallel to $<0.1$ arcsec while the energy selected from the synchrotron is varied by changing the Bragg angles. Furthermore, the design yields an exit beam whose offset with respect to the incident beam is independent of the chosen beam energy. Finally, the entire assembly was rotated about the incident-synchrotron-beam direction such that the plane formed by incident and diffracted beams was near $45^{\circ}$ degrees to the vertical.

The $\mathrm{x}$-ray beam from the compensator plate was allowed to fall upon a 1-mm-thick piece of Plexiglas after passing through a slit and ion chamber used for normalization purposes. A lithium-drifted silicon $\mathrm{Si}(\mathrm{Li})$ detector observed those $\mathrm{x}$ rays Compton scattered $90^{\circ}$ in the horizontal plane by the Plexiglas. The counting rate in the $\mathrm{Si}(\mathrm{Li})$ detector, normalized to the beam incident on the Plexiglas, as a function of beam energy chosen by the monochromator-polarizer system is plotted in Fig. 2. Strong oscillations, with increasing amplitude for increasing energy are noted. The horizontal scale in the plot is chosen in accord with Eq. (1) (see below) which results in the oscillatory behavior of the data being represented quite accurately by a damped sinusoidal wave. The reason for this will be described shortly.

The origin of the above oscillations, which we refer to as polarization Pendellösung, may be understood as follows. For the case of a single strong diffraction in

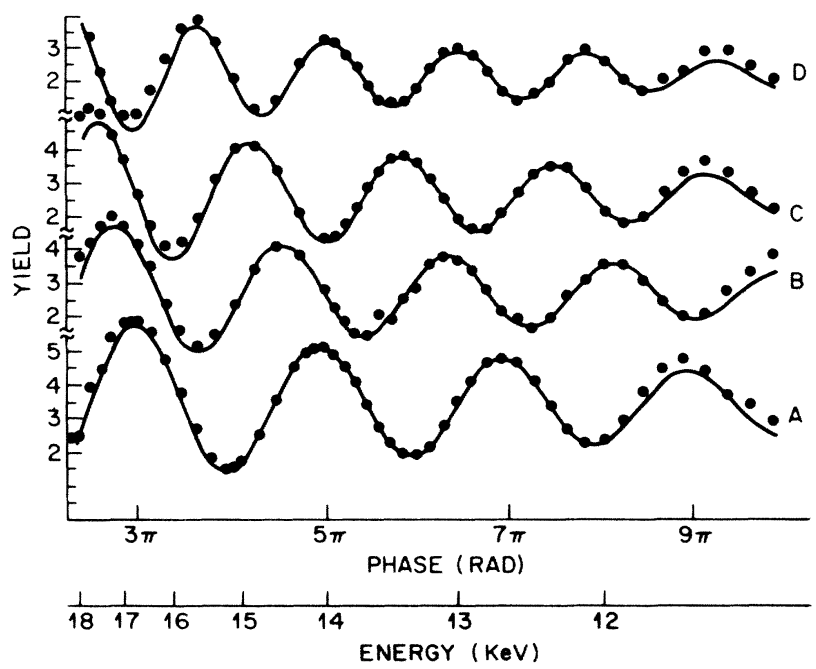

FIG. 2. Normalized yield in $\mathrm{Si}(\mathrm{Li})$ detector No. 1 vs energy of monochromator containing $\mathrm{x}$-ray compensator. Curves $A-D$ refer to the different compensator thicknesses. The phase assignment in the abscissa applies only to curve A. the Laue geometry, the eigensolutions to the Maxwell equations inside the crystal can be classified into two possible states of linear polarization. One of these, the solutions of the so-called sigma type, consists of running Bloch waves each composed of coupled plane waves, whose electric field vector is perpendicular to the plane formed by the incident- and diffracted-beam directions. For this transverse electric case, the polarization direction is parallel to the diffracting plane. The other class of polarization solutions, called pi, is transverse magnetic and consists again of coupled plane waves in the incident and diffracted directions but now the electric vector of each of these lies in the plane formed by the two directions. Therefore, the polarization can be considered as perpendicular to the diffracting plane.

For each state of polarization at fixed $x$-ray energy there are two kinds of $x$-ray Bloch-wave states. One, the so-called alpha-type solution, has standing-wave character perpendicular to the diffracting planes such that the field intensity has the same periodicity and a phase which places its nodes directly on the planes. The other type solution, called beta, is identical except that its antinodes are on the diffracting planes.

We now imagine an external plane wave impinging on the crystal at the exact Bragg condition with a polarization vector at $45^{\circ}$ to the diffracting planes. In order to match the internal- and external-wave solutions at the entry surface a linear combination of equal amounts of sigma and pi waves must be excited in the crystal, with each of these having equal amounts of alpha and beta character. The Poynting vector for each of these Bloch waves at the exact Bragg condition lies along the crystal planes so they all propagate in the same direction into the crystal.

We note that the alpha solutions have their nodal planes on the diffracting planes, which is where the majority of the strong $\mathrm{x}$-ray absorption takes place and hence these waves should propagate significantly farther through the crystal than the beta solutions, which have their maximum intensity on the planes. Hence, following the suggestion of $\mathrm{Hart},{ }^{2}$ our compensator plate has been cut thick enough so that only the alpha solutions survive to the back surface. Therefore, normal Pendellösung, which is basically a beating between alphalike and betalike solutions will have nothing to do with the oscillations with which this paper deals.

The effective index of refraction for the surviving two alpha solutions is slightly different (because the two solutions sample slightly different electron densities) leading to different phase velocities for the two waves. At the back surface of the plate, plane waves will be formed whose polarization state will depend upon the accumulated phase difference between the two internal eigenfunctions. Using the dynamical theory to account quantitatively for the effect one 
finds that the phase difference between the two solutions at the back of the plate is given by

$$
\delta=4 \pi^{3}\left(\frac{m c^{2}}{\alpha E}\right)^{3} \frac{\left|F_{G}\right|}{\cos \theta_{\mathrm{B}}} \frac{r_{e}^{4}}{d^{2} v} D,
$$

where $E$ is the $\mathrm{x}$-ray energy, $\alpha$ the fine-structure constant, $r_{e}$ the classical electron radius, $v$ the crystal unit-cell volume, $d$ the Miller-plane spacing, $D$ the crystal-plate thickness, $F_{\mathrm{G}}$ the crystal structure factor, and $\theta_{\mathrm{B}}$ the Bragg angle. For $\delta=2 \pi n$ with $n$ an integer it follows that the final state of polarization is the same as the initial state, i.e., plane polarized in the horizontal direction. For $\delta=2 \pi\left(n+\frac{1}{2}\right)$ the final state of polarization should be plane polarized in the vertical direction. Finally for $\delta=2 \pi\left(n+\frac{1}{4}\right)$ or $2 \pi\left(n-\frac{1}{4}\right)$ we expect left- and right-hand circularly polarized $\mathrm{x}$ rays, respectively, and hence the compensator becomes a quarter-wave plate.

Figure 2, curve $A$, shows the data for the $90^{\circ}$ Compton yield in the horizontal plane plotted as a function of beam energy and the derived phase $\delta$ from Eq. (1). The fitted curve corresponds to an exponentially damped sinusoidal function of $\delta$ with period $2 \pi$. The minima occur at $\delta=2 \pi n$ as they should for horizontally polarized $\mathrm{x}$ rays. The maxima at $\delta=2 \pi\left(n+\frac{1}{2}\right)$ represent vertical polarization. The states of circular polarization should occur halfway between the maxima and minima; verification of this state of polarization will be demonstrated shortly through the observation of magnetic Compton scattering. First, however, we note that it is possible to independently determine the number of "quarter waves" in the plate by changing the plate thickness and recording the shifted polarization Pendellösung fringes. This was accomplished experimentally by rotating the plate about an axis perpendicular to the diffracting planes. This changes only the effective plate thickness seen by the beam. Thus Fig. 2, curve $B$, obtained with a rotation of approximately $22^{\circ}$, Fig. 2 , curve $C$, with $32^{\circ}$, and Fig. 2, curve $D$, with $45^{\circ}$. Notice that at an energy of approximately $14 \mathrm{keV}$ increasing the plate thickness by $41 \%$ results in one additional polarization cycle. This agrees precisely with the phase assignment of $\delta=5 \pi$ for the peak in curve $A$ at $14 \mathrm{keV}$ and constitutes an independent measurement of the oscillation length inside the plate. Our final comments on the data in Fig. 2 deal with the degree of modulation in the oscillatory signals. Clearly the degree of polarization seems to be improving as the beam energy increases. The main reason for this feature lies in the fact that the asymmetric collimating crystal has its highest asymmetry, and hence its highest degree of angular collimation, at the highest energies in Fig. 2. Eigenfunctions in the quarter-wave plate excited by incident rays slightly off the exact Bragg condition do not have perfect nodes on the planes for the sigma polarization state and the difference between the effective index of refraction for sigma and pi polarization states is reduced. This results in a modified Pendellösung length as a function of incidence angle to the plate and a smearing of results at low energies as a result of beam divergence. In addition, no special precautions were taken in these preliminary experiments to minimize air scattering near the $\mathrm{Si}(\mathrm{Li})$ detector No. 1 and multiple scattering in the Plexiglas target, both of which contribute to reduced modulation in Fig. 2.

A magnetic-Compton-scattering experiment was performed to demonstrate directly the presence of circularly polarized $x$ rays. A beam energy of $17.5 \mathrm{keV}$ was chosen (nearly twice the critical energy where the incident synchrotron radiation is essentially completely horizontally polarized). The plate was adjusted to a thickness of $\frac{11}{4}$ Pendellösung waves (see curve $A$ in Fig. 2), and the transmitted $x$ rays were scattered from a magnetized iron target as shown in Fig. 1. The flux onto the sample was $-3 \times 10^{9}$ photons/sec, consistent with the expected $15 \%$ conversion efficiency from plane to circular polarization for the compensator plate. The Compton-scattering rate at a scattering angle of $145^{\circ}$ was monitored with a solid-state detector with the target and its immediate vicinity bathed in a helium atmosphere to avoid air-scattering background. Figure 3 shows the spectrum observed in the $\mathrm{Si}(\mathrm{Li})$ detector after a total of $9 \mathrm{~h}$ of data accumulation with the sample magnetized alternatively along and against the spin direction of the incident $x$ rays. The difference spectrum is also shown in the figure. The cross section is seen to differ by $0.5 \%$ for the two cases. ${ }^{13}$

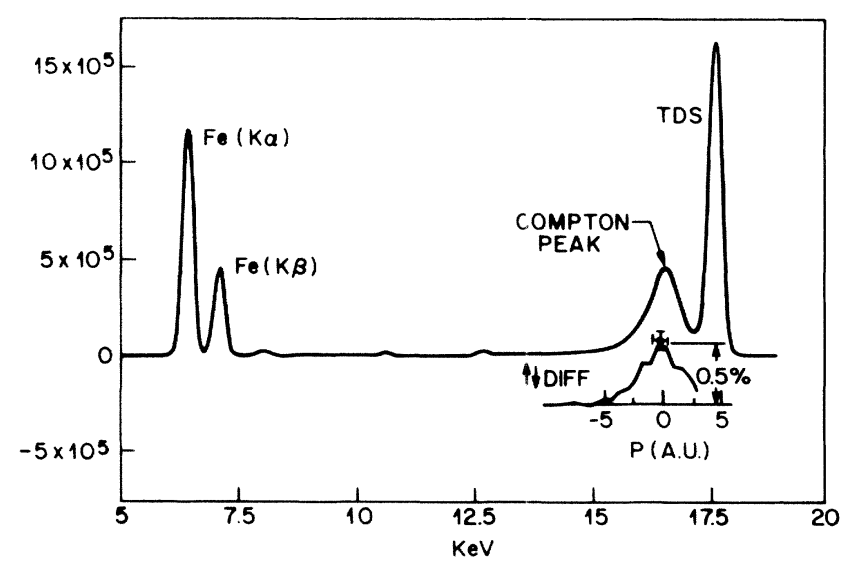

FIG. 3. Spectrum in $\mathrm{Si}(\mathrm{Li})$ detector No. 2 from magnetic-Compton-scattering experiment. Also shown is the difference spectrum described in the text. Vertical error beams are due to counting statistics and horizontal to detector resolution. The $\mathrm{Fe}$ fluorescence signals are spin independent and are used for accurate normalization in constructing the difference spectrum. 
The theoretically expected value ${ }^{14}$ is $0.75 \%$ for a $100 \%$ circularly polarized beam. The local abscissa for the difference spectrum in Fig. 3 has been converted to projected momentum as is customary in Comptonprofile studies of electron-momentum distributions. ${ }^{15}$ Unfortunately the poor relative resolution obtained by use of a $\mathrm{Si}(\mathrm{Li})$ detector at such low energies precludes our obtaining useful information on the contribution of conduction electrons to the magnetic moment of magnetized iron. This would require high resolution in the region below 1 a.u. which we have not obtained. It will therefore be necessary to perform similar experiments at higher energies $(>80 \mathrm{keV})$ where momentum resolution is greater or to develop higher-resolution detectors for use at lower energies.

The difference between the magnetic-scattering theory and this experiment is due to incomplete polarization of the beam. A density-matrix analysis of the compensator-prepared $x$-ray beam using the above two scattering experiments indicates that at the highest energy studied here the beam was $83 \%$ circularly polarized. Several of the reasons for lack of perfect polarization have already been discussed. In addition, strains in the crystal elements and alignment errors can contribute to a deterioration of performance. We hope to substantially improve the situation in future experiments.

In conclusion, we have succeeded in isolating the phenomenon of polarization Pendellösung using an $\mathrm{x}$ ray compensator plate in conjunction with continuous and linearly polarized radiation from a synchrotron source. The effect may be used to provide experimenters with a flexibility in choosing various states of photon polarization that is not currently available.
We gratefully acknowledge the contributions of the Cornell High-Energy Synchrotron Source staff to this work.

(a) Present address: Lawrence Livermore National Laboratory, Livermore, CA 94550.

(b) Present address: AT\&T Bell Laboratories, Whippany, NJ 07981.

1P. Skalicky and C. Malgrange, Acta Crystallogr., Sect. A 28, 501 (1972).

${ }^{2}$ M. Hart, Philos. Mag. B 38, 41 (1978).

${ }^{3}$ M. Hart and A. R. D. Rodriquez, Philos. Mag. B 40, 149 (1979).

${ }^{4} \mathrm{O}$. Brummer, Ch. Eisenschmidt, and H. R. Hoche, Z. Naturforsch. Teil A 37, 524 (1982).

5P. M. Platzman and N. Tzoar, Phys. Rev. B 2, 3556 (1970).

${ }^{6}$ N. Sakai and K. Ono, Phys. Rev. Lett. 37, 351 (1976).

${ }^{7}$ N. Sakai and K. Ono, J. Phys. Soc. Jpn. 42, 770 (1977).

${ }^{8}$ B. M. Kincaid, J. Appl. Phys. 48, 2684 (1977).

${ }^{9}$ K. J. Kim, Nucl. Instrum. Methods 219, 425 (1984).

${ }^{10}$ L. Azaroff, R. Kaplow, N. Kato, R. Weiss, A. Wilson, and R. Young, X-Ray Diffraction (McGraw-Hill, New York, 1974). Note the Pendellösung effect shown in Fig. 2 of Ref. 4 is not of the kind we discuss here.

${ }^{11}$ M. Renninger, Z. Naturforsch. Teil A 16, 1110 (1961).

12J. A. Golovchenko, R. A. Levesque, and P. L. Cowan, Rev. Sci. Instrum. 52, 509 (1981).

${ }^{13}$ Changing the sign of the circular polarization by adjusting the compensator changed the sign of the effect as expected.

${ }^{14}$ M. Bernardini, P. Brovetto, S. Debenedetti, and S. Ferroni, Nuovo Cimento 7, 416 (1958).

${ }^{15}$ Compton Scattering, edited by B. Williams (McGraw-Hill, New York, 1977). 


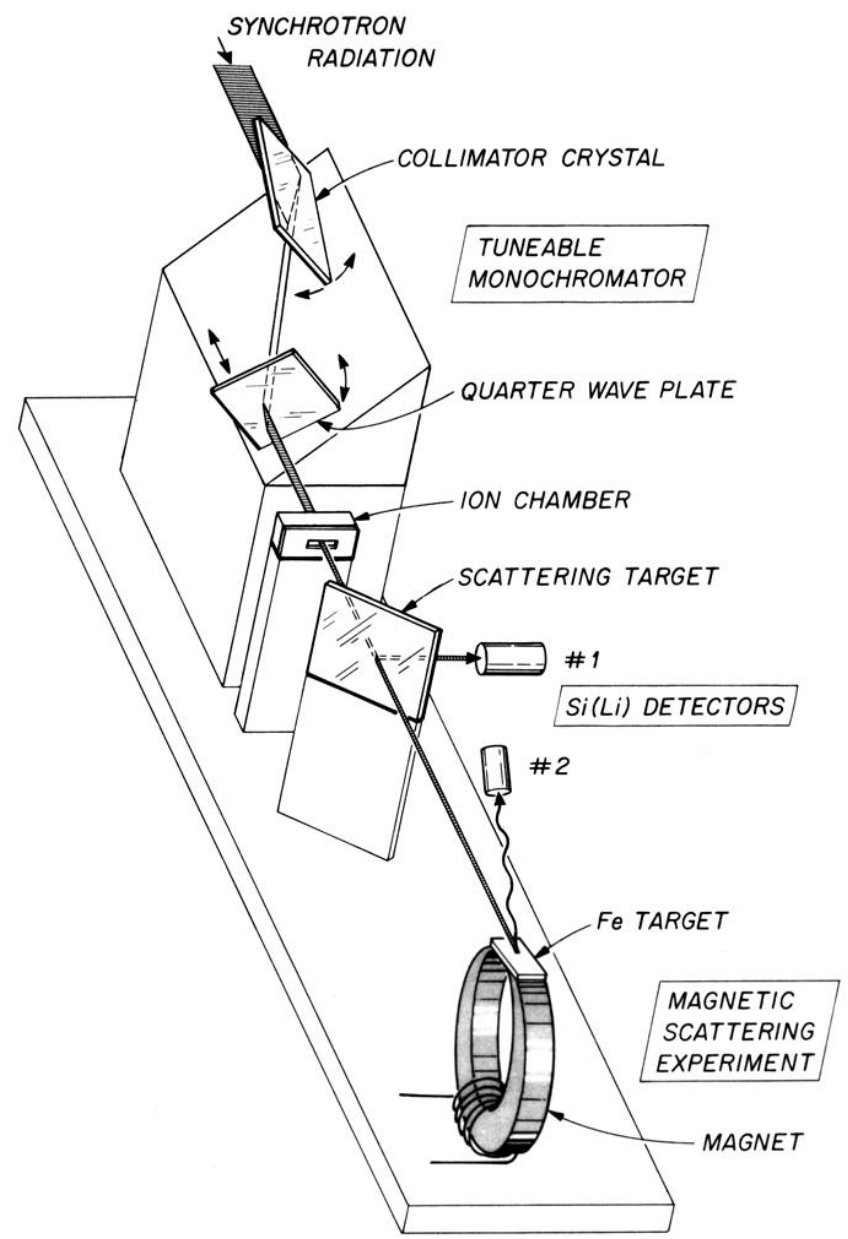

FIG. 1. Schematic layout of experiment. 